\title{
Variable Aperture Method of Ultrasonic Annular Array for the Detection of Addictive Manufacturing Titanium Alloy
}

\author{
Wenchao Li $\mathbb{D},{ }^{1}$ Junjie Chang $\mathbb{D},{ }^{1}$ Wentao $\mathrm{Li} \mathbb{D},{ }^{2}$ and Xiaoyun Long $\mathbb{C}^{3}$ \\ ${ }^{1}$ Key Lab of Nondestructive Testing, Ministry of Education, Nanchang Hangkong University, Nanchang 330063, China \\ ${ }^{2}$ School of Mechanical and Electronical Engineering, Lanzhou University of Technology, Lanzhou 730050, China \\ ${ }^{3}$ College of Information Engineering, Nanchang University, Nanchang 330031, China
}

Correspondence should be addressed to Junjie Chang; changjunjie_nhu@126.com

Received 18 November 2020; Revised 14 December 2020; Accepted 16 December 2020; Published 31 December 2020

Academic Editor: Bin Gao

Copyright (c) 2020 Wenchao Li et al. This is an open access article distributed under the Creative Commons Attribution License, which permits unrestricted use, distribution, and reproduction in any medium, provided the original work is properly cited.

\begin{abstract}
The ultrasonic annular array transducer usually has a stronger focusing acoustic field than the linear array and matrix transducer with the same number of array elements, and is more suitable for the detection of large thickness and high attenuation components. However, due to the special arrangement of array elements, the focusing beam cannot be deflected and has a large near field, which limits its application in practical detection. The element parameters of annular array transducer are often designed and analyzed according to the 2-D acoustic field model of a linear array transducer. Therefore, the 3-D acoustic field distribution model of the annular array transducer is established, and the influence of the element parameters on its spatial acoustic field focusing characteristics is analyzed. The design criteria of the array element division mode and element size are proposed, which can avoid the generation of high-energy side lobe and grating lobe, and have good axial acoustic field. Then, the influence of excitation aperture on the energy and size of focal spot at different depths is discussed. The dynamic focusing method with variable aperture of annular array is established, and the C-scan detection experiment is carried out on the addictive manufacturing titanium alloy specimen. The detection results show that the variable aperture method has better central amplitude consistency and imaging accuracy for different depth defects, and has better near surface detection ability than the fixed aperture method.
\end{abstract}

\section{Introduction}

Ultrasonic array technology is a kind of multichannel ultrasonic testing technology which arranges several piezoelectric wafers into an array according to a certain combination mode. By controlling the excitation sequence and delay time of piezoelectric wafers, the deflection and focusing of synthetic acoustic beam can be realized. At present, ultrasonic array transducers commonly used in industry can be divided into 1-D linear array, 1.5-D annular array, 2-D matrix array, etc. according to the element arrangement mode [1]. At present, ultrasonic the linear array transducer and ultrasonic matrix transducer are the most widely used in theoretical research and industrial application. The ultrasonic matrix array transducer can realize 3-D imaging [2], but the acoustic beam control algorithm is complex, and the manufacturing process and equipment hardware costs are high [3-5]. The ultrasonic linear array transducer is easier to manufacture, and its transmitting and receiving delay control method is relatively simple, which is mostly used in practical applications. However, the $\mathrm{C}$-scan detection results of ultrasonic linear array transducer are affected by its focal spot asymmetry, and the shape quantitative error of defects in ultrasonic linear array C-scan results is large [6]. The single element size of ultrasonic annular array transducer is larger, and it can achieve stronger focusing energy than ultrasonic linear array transducer with fewer array elements [7]. Meanwhile, its focal spot is completely symmetrical along the radial direction. It is an effective method to solve the problem of low signal-to-noise ratio (SNR) and large defect distortion in Cscan testing results of large thickness and high attenuation materials. However, due to the fact that the acoustic beam of ultrasonic annular array can only focus along the central axis, the beam cannot be deflected and the near field is large, 


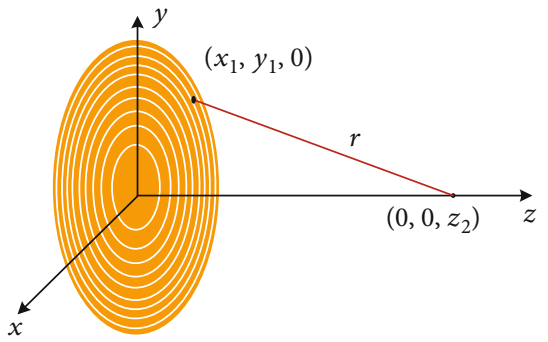

Figure 1: Calculation diagram of acoustic field of annular transducer.

so there is little research and application in the industrial field, and the design and verification method of the parameters and focusing algorithm of the ultrasonic annular array transducer are insufficient.

Firstly, based on the 3-D acoustic field calculation theory, the influence of the parameters of the ultrasonic annular array transducer on the focusing acoustic field characteristics is analyzed, and the design criteria of the detection parameters suitable for the annular array transducer are proposed. Then, the influence of focusing depth and excitation aperture size on the focal spot size and acoustic energy of annular array transducer is analyzed, and a variable aperture dynamic focusing method of annular array is established. The C-scan experimental results of additive titanium alloy show that the variable aperture dynamic focusing method can improve the detection ability and sensitivity of different depth defects. It provides theoretical guidance for the application of ultrasonic annular array transducer in the detection of large thickness and high attenuation components.

\section{Influence of Transducer Parameters on Acoustic Field}

2.1. Focusing Acoustic Field of Annular Array. Different from the acoustic field calculation of ultrasonic linear array transducer, only calculating the 2-D cross-sectional acoustic field along the array direction cannot accurately reflect the real focusing acoustic field of annular array transducer [8]. It is necessary to calculate the superimposed acoustic field in 3$\mathrm{D}$ space after the circular array element is discretized at a certain angle along the circumference. Firstly, the physical state relationship between any two points in space is established according to the transformed wave equation [9]:

$$
\int_{S_{\mathrm{T}}}\left(\overline{P_{2}} \frac{\partial \overline{P_{1}}}{\partial n}-\overline{P_{1}} \frac{\partial \overline{P_{2}}}{\partial n}\right) d S_{\mathrm{T}}=\int_{V}\left(\overline{P_{1}} \overline{f_{2}}-\overline{P_{2}} \overline{f_{1}}\right) d V
$$

Here, $P_{1}$ and $P_{2}$ are the acoustic pressures of two points in the medium at a certain time, $f$ is the body force, $S_{\mathrm{T}}$ is the element area of the transducer, and $V$ is the outside of the whole boundary of the array element. To calculate the acoustic field distribution in space, Green's function $\bar{G}(x ; y$

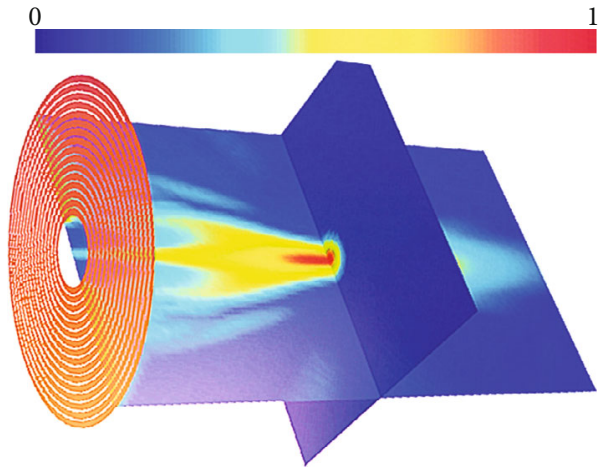

Figure 2: The focusing acoustic field of ultrasonic annular array transducer.

, $\omega)$ in 3-D space should be solved firstly:

$$
\bar{G}(x ; y, \omega)=\frac{\exp (-j k r)}{4 \pi r},
$$

where $k$ is the wavenumber and $r$ is the distance from a coordinate point to a fixed point in 3-D space.

As shown in Figure 1, it is expressed as the distance from any point of transducer to any point on the central axis, i.e., $r=\sqrt{x_{1}^{2}+y_{1}^{2}+z_{2}^{2}}$.

Assuming that $\delta(x-y)$ is the shock response function and $\bar{G}(x ; y, \omega)$ is the acoustic solution of the function as a body force $f$, the two solutions of the wave equation can be assumed as follows:

$$
\begin{gathered}
\left\{\begin{array}{l}
\bar{P}_{1}(x, \omega)=\bar{P}(x, \omega), \\
\bar{f}_{1}(x, \omega)=0,
\end{array}\right. \\
\left\{\begin{array}{l}
\bar{P}_{2}(x, \omega)=\bar{G}(x ; y, \omega), \\
\bar{f}_{2}(x, \omega)=\delta(x-y) .
\end{array}\right.
\end{gathered}
$$

Take Equation (3) into Equation (1), and then express it as follows:

$$
\bar{P}(y, \omega)=\int_{S}\left[\bar{G}(y ; x, \omega) \frac{\partial \bar{P}(y, \omega)}{\partial n}-\bar{P}(y, \omega) \frac{\partial \bar{G}(y ; x, \omega)}{\partial n}\right] d S .
$$

Combined with the deformation formula $\bar{P}=-j \omega \rho_{0} \bar{\varphi}$ of Newton's second law and Equation (2), for any point in the radiation space, the total acoustic pressure of the synthetic beam can be obtained by adding the acoustic pressure of $N$ elements with the following equation.

$$
p(x, t)=\sum_{i=1}^{N} \sqrt{\frac{c}{t}} \otimes \int_{S_{\mathrm{T}}} \frac{V_{n}(t-(r / c))}{4 \pi r} d S_{\mathrm{T}}(t),
$$

where $V_{n}$ is the excitation signal of the array element, $x$ and $t$ represent the spatial position and time, respectively, $c$ is the 


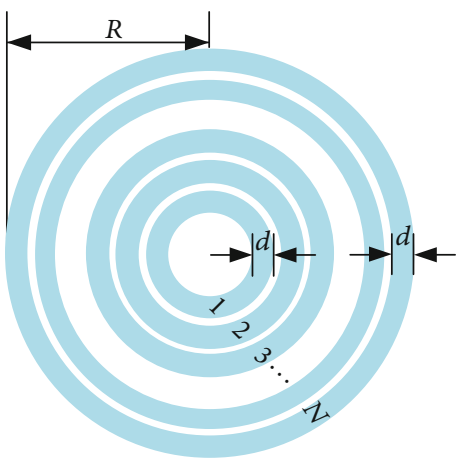

(a)

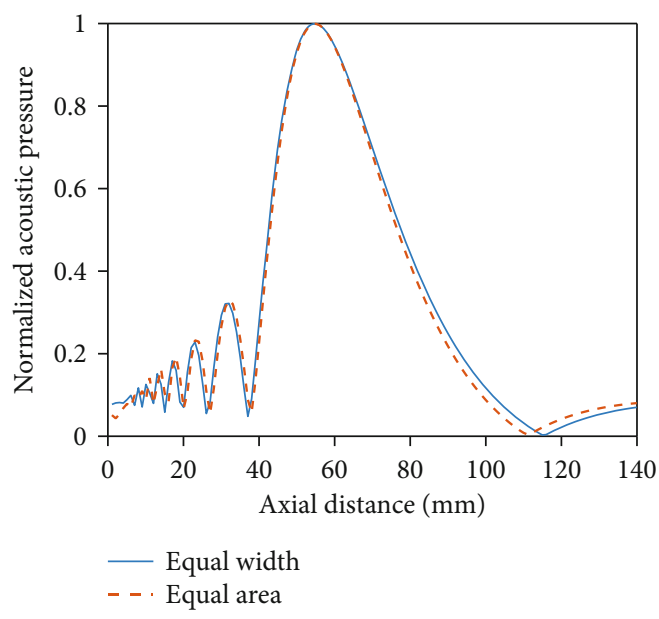

(c)

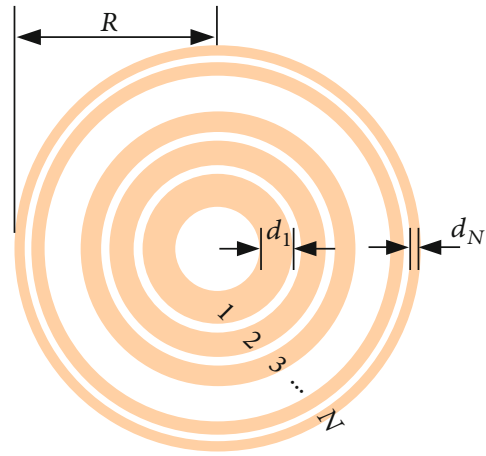

(b)

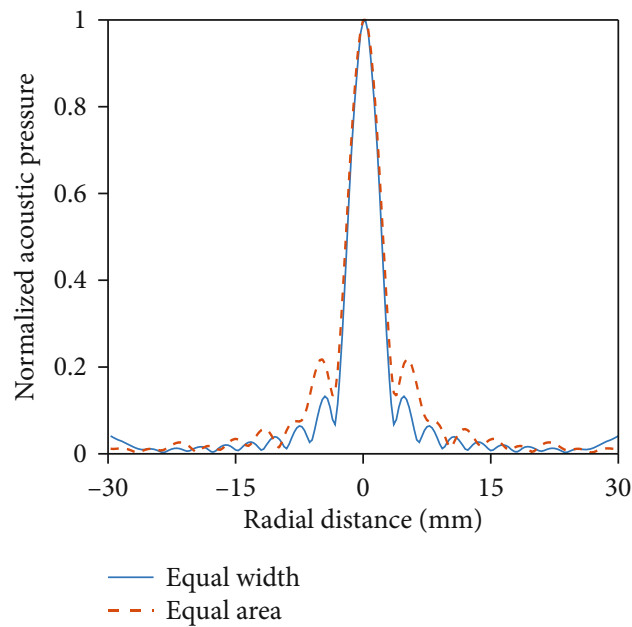

(d)

Figure 3: Acoustic pressure distribution of two types of annular array elements. (a) The element with equal width. (b) The element with equal area. (c) Axial acoustic field distribution. (d) Radial acoustic field distribution.

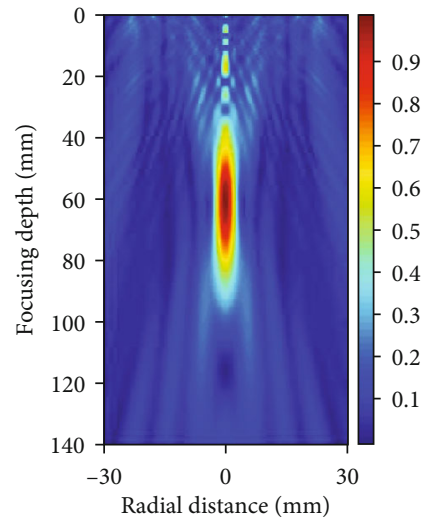

(a)

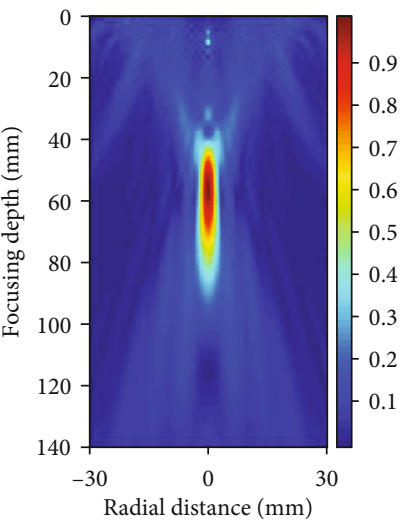

(b)

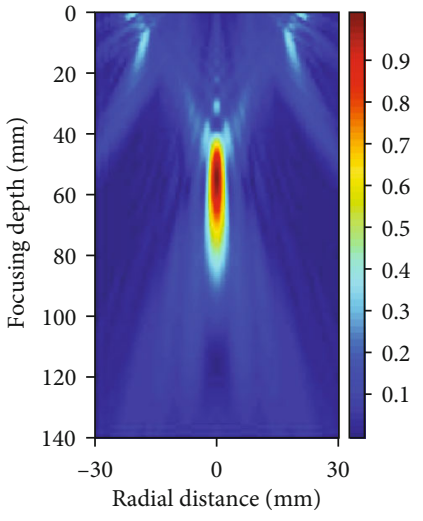

(c)

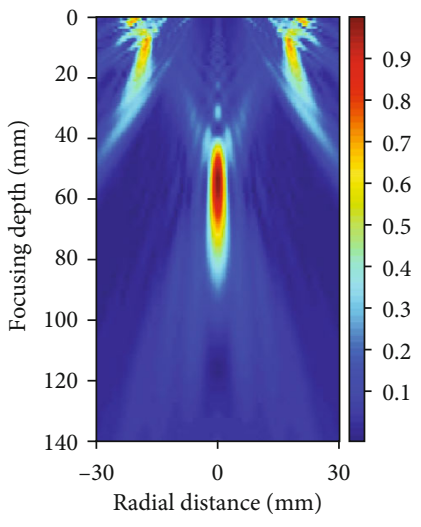

(d)

Figure 4: Focusing acoustic field distribution of annular array with different array element sizes. (a) $N=4, d=2.6 \mathrm{~mm} \approx 4 \lambda$. (b) $N=8, d$ $=1.3 \mathrm{~mm} \approx 2 \lambda$. (c) $N=16, d=0.65 \mathrm{~mm} \approx \lambda$. (d) $N=32, d=0.325 \mathrm{~mm} \approx 0.5 \lambda$.

sound velocity of the medium, and $S_{\mathrm{T}}$ is the array element's area $[10,11]$.

The spatial focusing acoustic field of the ultrasonic annular array transducer is shown in Figure 2.
2.2. Selection and Design Method of Array Element Parameters. When the total element area is fixed, the annular array transducer element can be divided into two types: the element with equal width and the element with equal area, 


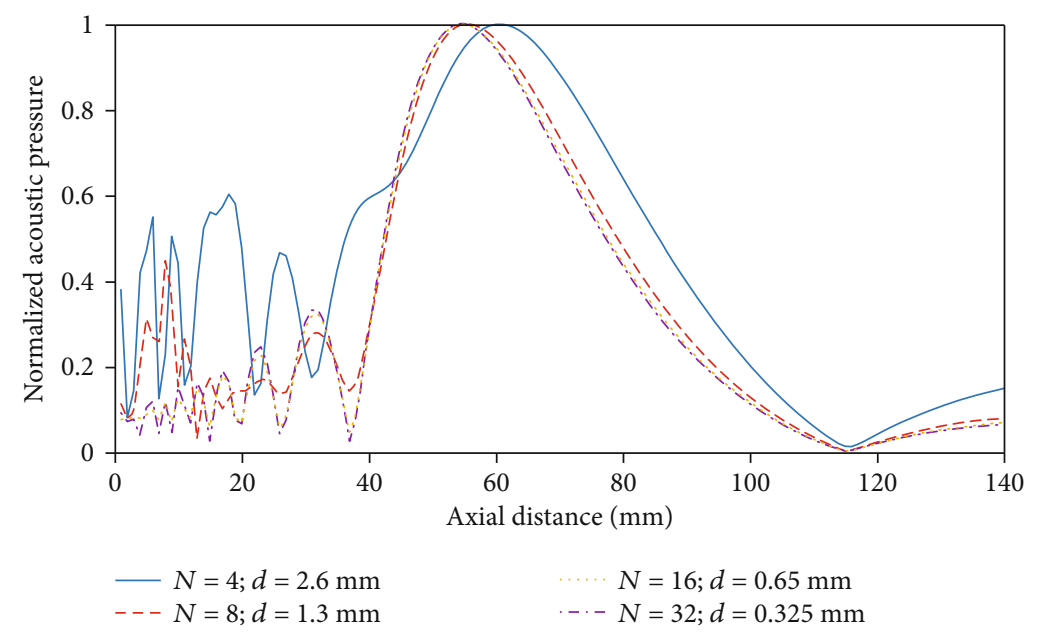

FIgURE 5: Axial acoustic pressure of annular array with different array element sizes.

TABLE 1: Simulation parameters of ultrasonic annular array.

\begin{tabular}{ccccc}
\hline No. & $\begin{array}{c}\text { Element pitch } \\
(\mathrm{mm})\end{array}$ & $\begin{array}{c}\text { Focusing depth } \\
(\mathrm{mm})\end{array}$ & $\begin{array}{c}\text { Resolution } \\
(\mathrm{mm})\end{array}$ & $\begin{array}{c}\text { Focusing } \\
\text { method }\end{array}$ \\
\hline 1 & 0.65 & $20-120$ & 0.1 & $\begin{array}{c}\text { Fixed } \\
\text { aperture } \\
(16)\end{array}$ \\
2 & 0.65 & $20-120$ & 0.1 & $\begin{array}{c}\text { Fixed } F / D \\
(\sim 4.4)\end{array}$ \\
3 & 0.65 & $20-120$ & 0.1 & $\begin{array}{c}\text { Fixed } F / N_{\mathrm{f}} \\
(\sim 0.5)\end{array}$ \\
\hline
\end{tabular}

as shown in Figure 3(a) and Figure 3(b). The area of the element increases with the outer element from the inner element under the partition form of equal element width. The width of the outer element will decrease exponentially with the increase of the number of elements when the area of each element is equal. Figure 3(c) and Figure 3(d) show the acoustic pressure distribution at the depth of $60 \mathrm{~mm}$ of the annular array transducer with 12 elements of the equal width and equal area array elements. It can be seen that the difference of axial acoustic field distribution between the two array element partition methods is very small, and the radial focal spot size is basically the same. However, when the number of array elements required is large, the width difference between the outer element and the inner element will be too large, leading to the difficulty of micromachining.

The number and width of the elements can be directly affected by the size of the elements when the total area of the circular array element is fixed. The influence of different element sizes on the focusing acoustic field of annular array transducer is analyzed to establish the design criteria of element parameters. Figure 4 shows the 2-D cross-sectional simulation results of $3-\mathrm{D}$ focusing acoustic field distribution of annular array with different array element partition sizes.

Due to the equal area of the total array elements, the focal spot sizes are close to each other under different element sizes [12]. However, different from the design criteria for the elements of ultrasonic linear array transducer, the ultrasonic annular array transducer still has strong main lobe energy at $60 \mathrm{~mm}$ focal depth when there are only $N=4$ elements. The influence of element width on the side lobe and grating lobe of focusing acoustic field is also different from that of ultrasonic linear array transducer: When the element width $d$ is close to half wavelength, as shown in Figure 4(d), there are strong grating lobes on both sides of the transducer. However, the side lobe with higher energy will be produced when the element width $d \approx 4 \lambda$, as shown in Figure 4 (a). Figure 4(b) and Figure 4(c) show that when the element width of the ultrasonic annular array transducer is between the wavelength and twice the wavelength, that is, when $\lambda \leq$ $d \leq 2 \lambda$, the side lobe and grating lobe with higher energy can be avoided at the same time. In addition, the axial acoustic field distribution characteristics under different array element sizes are further analyzed to better realize the full depth range detection, as shown in Figure 5.

The energy and size of the main lobe of the focusing beam are mainly determined by the element number when the total array area is fixed. According to the results of the axial acoustic pressure, the focal spot sizes of the three results are consistent when the number of elements $N \geq 8$. For the axial acoustic pressure in the nonfocusing region, the peak value of side lobe has reached $50 \%$ of the peak value of the main lobe in the initial $20 \mathrm{~mm}$ depth when $d=2.6 \mathrm{~mm} \approx 4 \lambda$ and $d=1.3 \mathrm{~mm} \approx 2 \lambda$. When $d=0.65 \mathrm{~mm} \approx \lambda$ and $d=0.325$ $\mathrm{mm} \approx 0.5 \lambda$, the acoustic pressure fluctuation in the nearfield region is relatively stable. In conclusion, when the element width $d \approx \lambda$, the ultrasonic annular array transducer has the best overall focusing acoustic field characteristics, which can avoid the generation of high-energy side lobe and grating lobe, and has better axial acoustic field.

\section{Variable Aperture Focusing Detection Method}

3.1. Theory Analysis. The distribution of the focusing acoustic field of the ultrasonic annular array transducer is like that of the single crystal disc with different focal length lenses, that is, it satisfies the ultrasonic focusing theory of the disk 


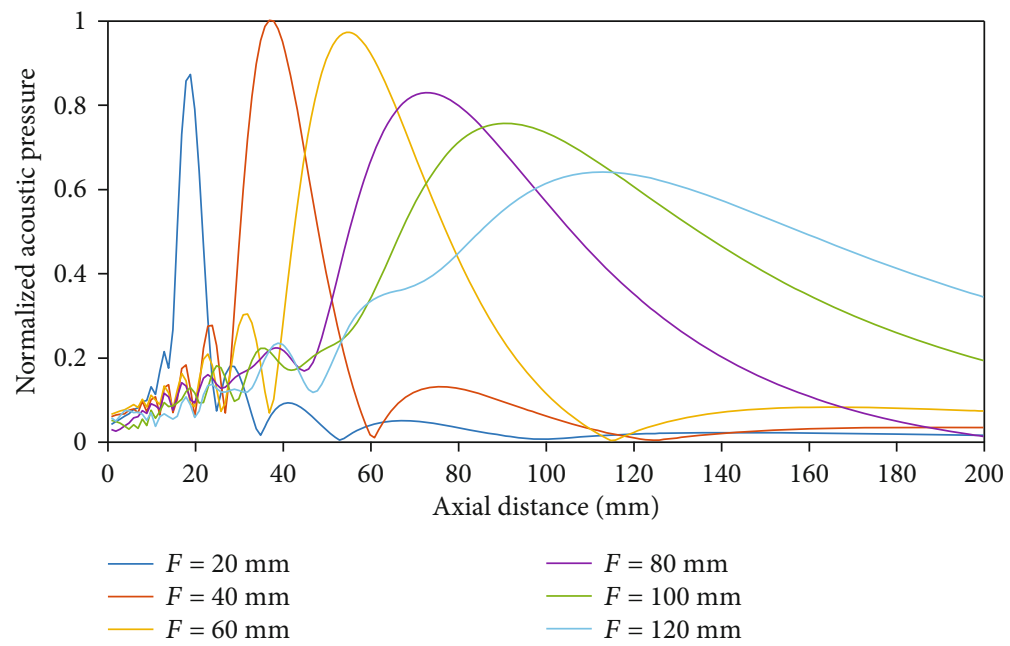

(a)

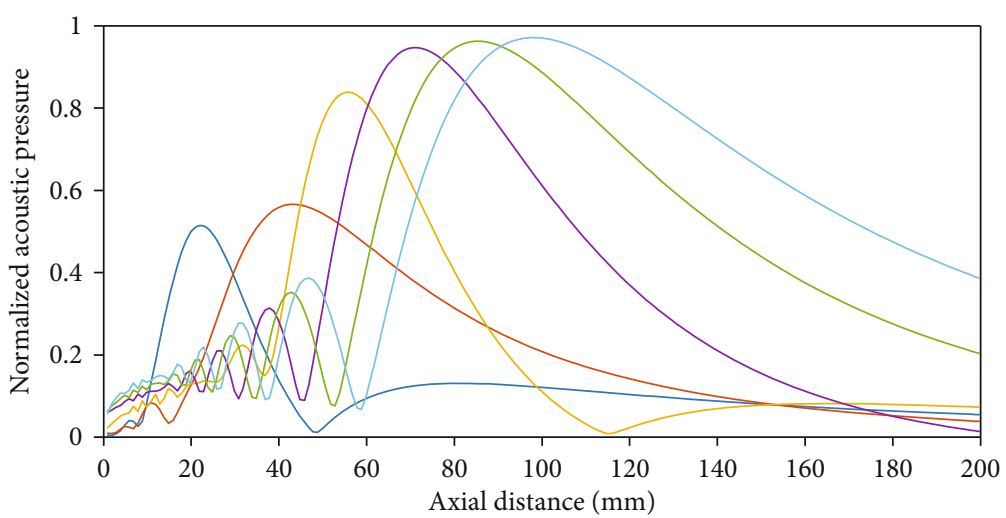

$$
\begin{aligned}
& -F=20 \mathrm{~mm} \\
& F=40 \mathrm{~mm} \\
& F=60 \mathrm{~mm} \\
& \text { - } F=80 \mathrm{~mm} \\
& \text { - } F=100 \mathrm{~mm} \\
& F=120 \mathrm{~mm}
\end{aligned}
$$

(b)

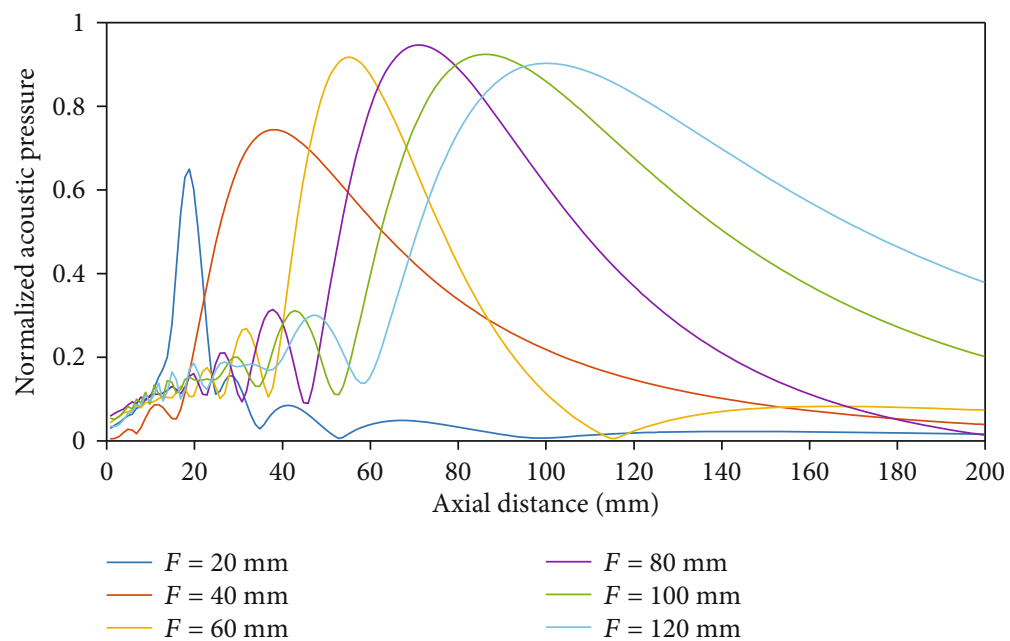

(c)

Figure 6: Axial acoustic field distribution of annular array with different aperture excitation methods. (a) Fixed aperture method. (b) Variable aperture method for fixed $F / D$. (c) Variable aperture method for fixed $F / N_{\mathrm{f}}$. 


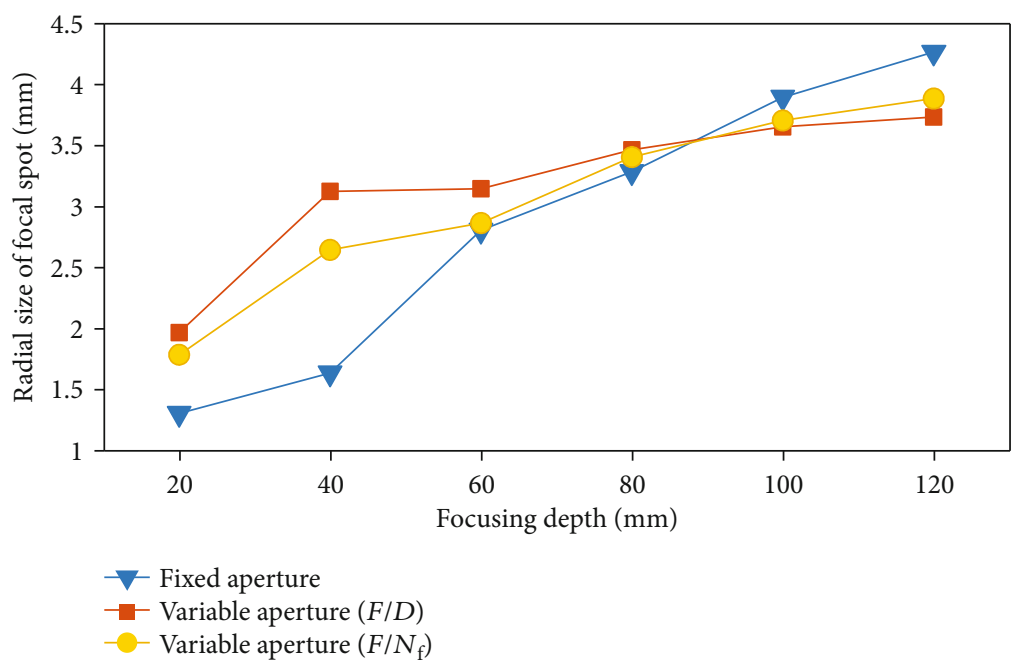

FIgURE 7: Variation of radial size of focal spot with different focusing depths.
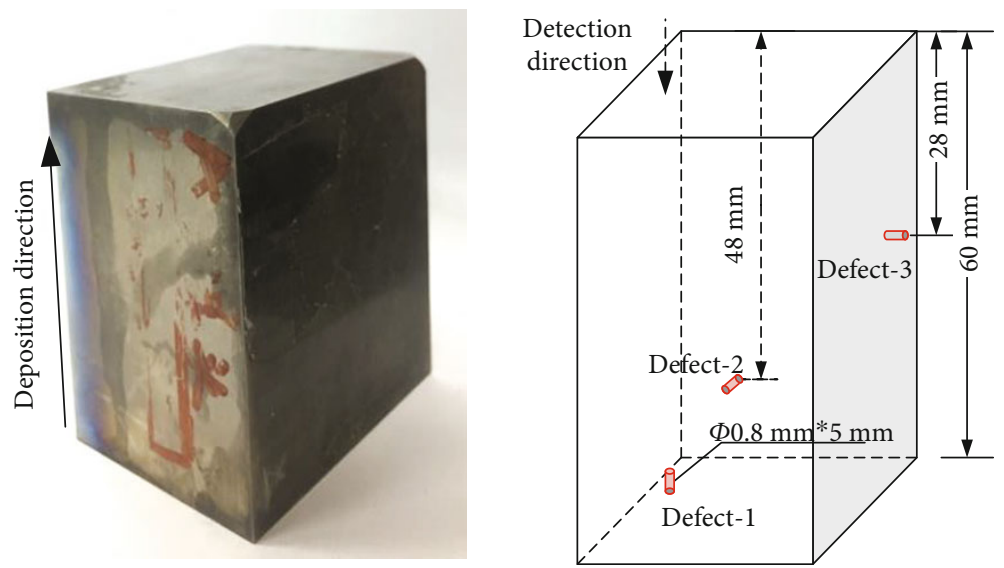

FIGURE 8: Test specimen of additive manufacturing with prefabricated defects.

acoustic field [13]. The width of the focusing beam is measured by $-6 \mathrm{~dB}$ method as the focal spot diameter, and the focal spot diameter of the focusing beam can be expressed as follows:

$$
\Phi=\Phi_{-6 \mathrm{~dB}} \approx \frac{\lambda F}{D}
$$

where $\lambda$ is the wavelength, $F$ is the focal length, and $D$ is the diameter of the disc.

Therefore, when the wavelength is constant, the similar focal spot diameter can be obtained in different depth regions by fixing the ratio of focal length to element diameter of annular array transducer. However, this fixed focal spot diameter variable aperture method will lead to changes in the near-field length and focusing energy. Therefore, the variable aperture method can also be established by fixing the focusing intensity and the near-field ratio [14]. According to the near-field formula and the intensity of focusing acoustic field, the approximate characterization methods are as fol- lows:

$$
\left\{\begin{array}{l}
N_{\mathrm{f}}=\frac{D^{2}}{4 \lambda}, \\
I \approx\left[\frac{\pi}{2} \times\left(\frac{F}{N_{\mathrm{f}}}\right)\right]^{2},
\end{array}\right.
$$

where $N_{\mathrm{f}}$ is the near-field length of the transducer and $I$ is the relative intensity of the focusing acoustic field.

It can be seen that the intensity of focusing acoustic field at different depths is relatively consistent when the ratio of focal length to near-field length is fixed. Therefore, two different variable apertures focusing detection methods can be established, namely, fixed $F / D$ and $F / N_{\mathrm{f}}$.

3.2. Analysis of Focusing Acoustic Field Characteristics. In the simulation model, the focusing acoustic field is distributed along the axial direction in the depth range of $0-120 \mathrm{~mm}$, and a total of 6 focusing points are set at the interval of $20 \mathrm{~mm}$. The excitation elements of the fixed aperture method are all set as 16 elements. In order to ensure the dynamic 


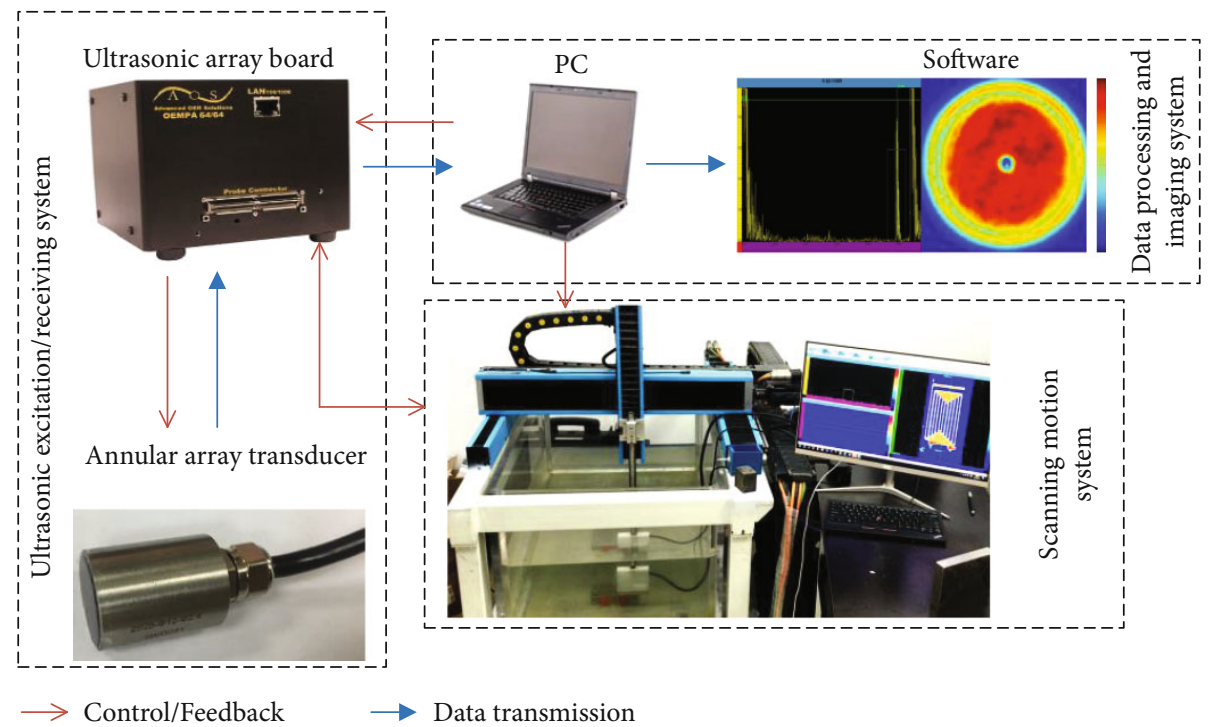

(a)

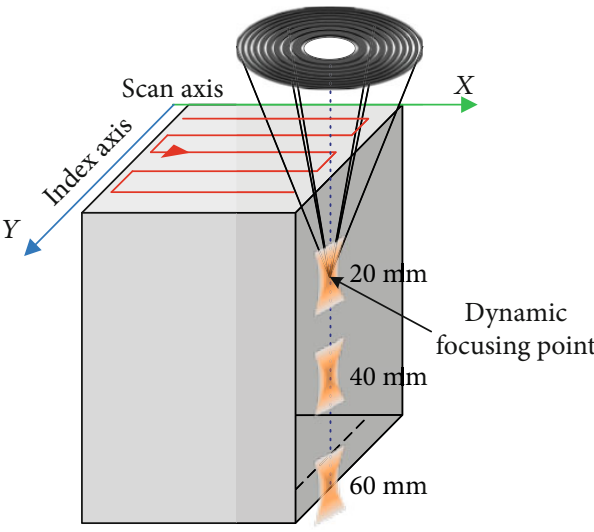

(b)

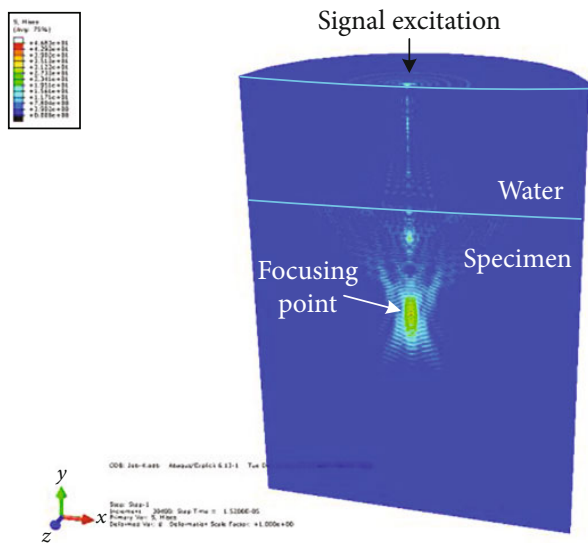

(c)

FIgURE 9: Experimental setup of ultrasonic annular array detection system. (a) Main components of detection system. (b) Schematic of the dynamic focusing method using the annular array. (c) The 3D FEM model of acoustic beam propagation of annular array in double-layer medium.

focusing effect, the minimum excitation elements of the two variable aperture methods are not less than 4 . By changing the number of excitation array elements, the scale factor of the two variable aperture methods is kept unchanged as far as possible. The specific simulation parameters are shown in Table 1.

The axial acoustic field distribution of the three focusing methods at different depths is shown in Figure 6. It is shown in Figure 6(a) that the focusing method of annular array with fixed aperture has higher focusing beam energy in the focusing depth of $20-80 \mathrm{~mm}$. However, with the increase of the focusing depth, the axial size of the focal spot increases obviously, and the focusing energy decreases rapidly, with the maximum difference of nearly $40 \%$. In addition, the focal spot length of the near surface is small under this detection method, and when the dynamic focusing points are spaced at a large distance, the nonfocusing region (such as $30 \mathrm{~mm}$ in Figure 6(a)) may become a detection blind zone. The simulation results of the fixed $F / D$ variable aperture method are shown in Figure 6(b). Different from the fixed aperture focusing method, the axial size difference of the focal spot at a larger focusing depth $(>40 \mathrm{~mm})$ is smaller. However, the energy of focal spot field is lower at a smaller focusing depth, and the difference between the maximum and minimum amplitude of each focusing center is about $47 \%$. Figure 6(c) shows the simulation results of the fixed $F / N_{\mathrm{f}}$ variable aperture method. It can be found that the difference of energy and length of focal spot is the smallest at different focusing points. The maximum difference of central amplitude of all focusing center is about $31 \%$, and the difference of central amplitude is less than $5 \%$ in the detection range of 60-120 mm, which can ensure more consistent detection sensitivity for different depth defects of the component. In addition, the acoustic pressure of the variable aperture method for fixed $F / N_{\mathrm{f}}$ is increased obviously in the nonfocusing region with the depth of $30 \mathrm{~mm}$, which is about 3 times of the fixed aperture method and 2 times of the variable aperture method for fixed $F / D$. Therefore, the variable 


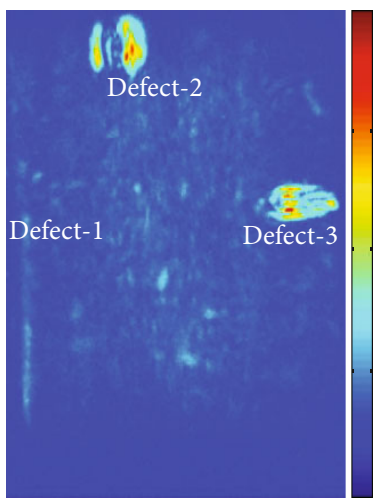

(a)

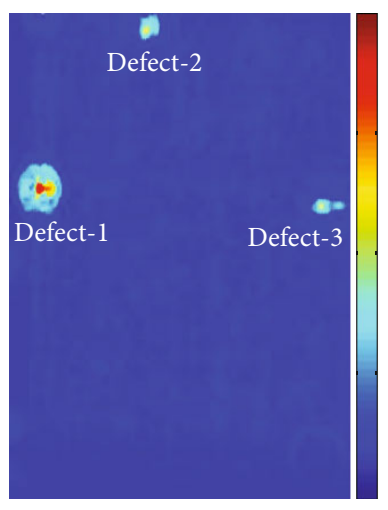

(b)

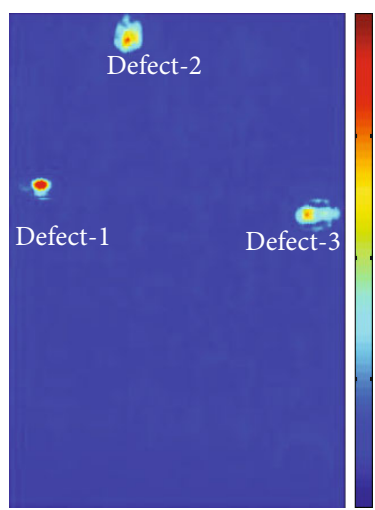

(c)

FIGURE 10: C-scan results of the additive manufacturing specimen of dynamic focusing method. (a) Fixed aperture method of linear array (10 MHz, 64 elements). (b) Fixed aperture method of annular array. (c) Variable aperture method of annular array.

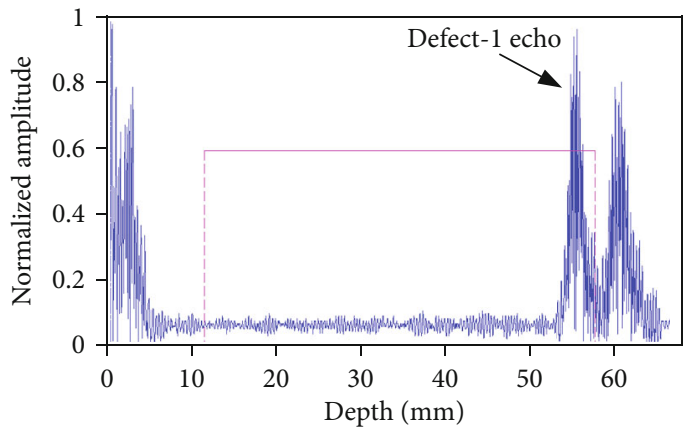

(a)

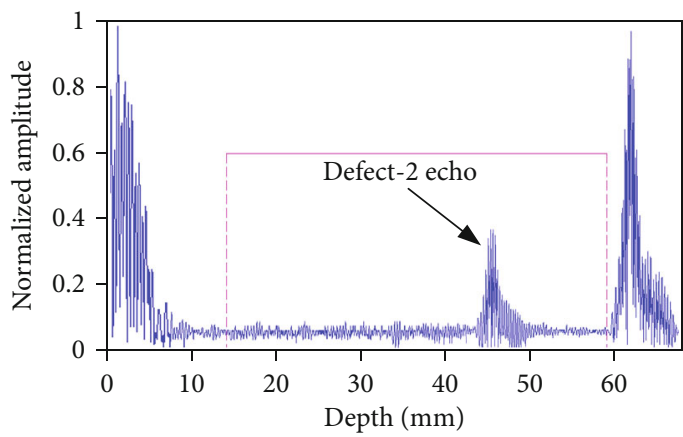

(c)

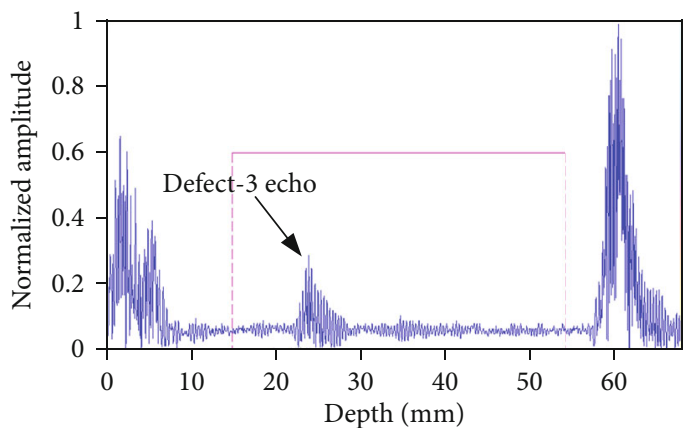

(e)

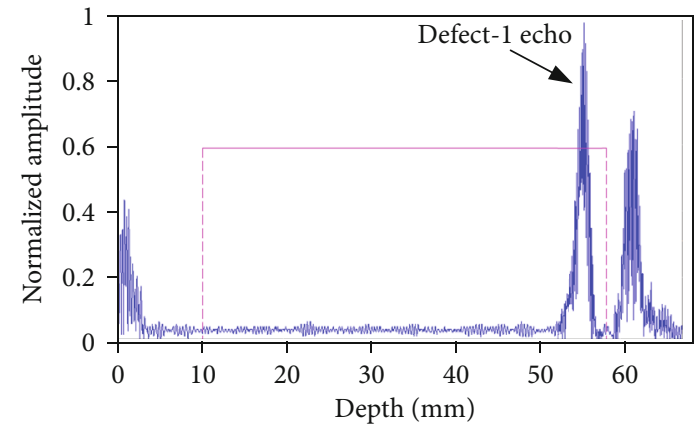

(b)

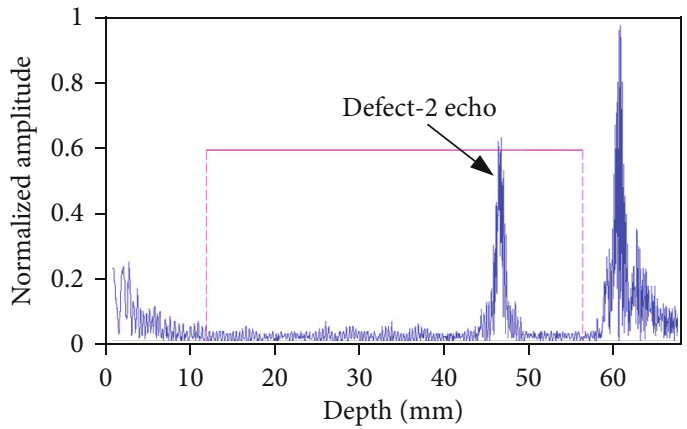

(d)

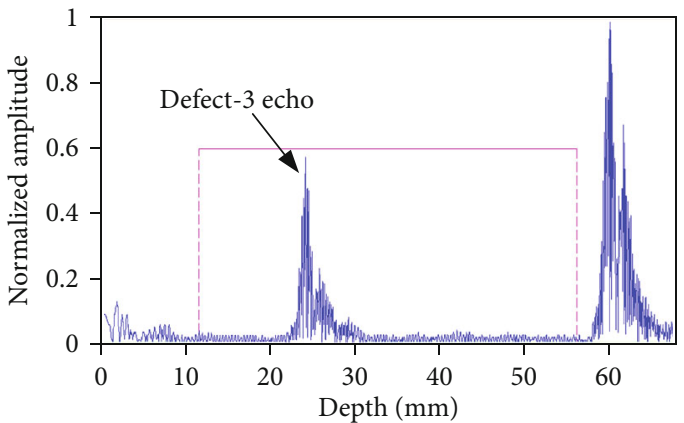

(f)

FIGURE 11: Echo signal of the defects using annular array. (a, c, e) Echo signals of defect-1, defect-2, and defect-3 using the fixed aperture method, respectively. (b, d, f) Echo signals of defect-1, defect-2, and defect-3 using the variable aperture method, respectively. 
aperture method for fixed $F / N_{\mathrm{f}}$ can avoid the problem of missing detection caused by the large spacing of the focusing points.

The axial distribution characteristics of acoustic field will affect the detection sensitivity and maximum detection depth, while the radial distribution characteristics of acoustic field will directly affect the quantitative accuracy of C-scan inspection results $[15,16]$. The $-6 \mathrm{~dB}$ method is used to measure the radial size of the focal spot of the fixed aperture method and the two variable aperture methods. The variation of the radial size of the focal spot with the focusing depth in the range of $20-120 \mathrm{~mm}$ is shown in Figure 7.

The measurement results show that the radial size of the focal spot changes mostly with the increase of the focusing depth under the fixed aperture focusing method, and the difference between the maximum value and the minimum value in the range of $20-120 \mathrm{~mm}$ is about 3.3 times. The fluctuation of focal spot size of the two variable aperture focusing methods is better than that of the fixed aperture focusing method. The change range of the focal spot size of the fixed $F / D$ variable aperture focusing method is the smallest, the difference between the maximum and the minimum is about 1.9 times, and the change range of focal spot size is less than $0.7 \mathrm{~mm}$ when the depth is more than $40 \mathrm{~mm}$. The simulation results show that the axial and radial acoustic field distribution characteristics of the variable aperture focusing method are better than those of the fixed aperture focusing method. For different depth focusing points, the fixed $F / D$ variable aperture method has the best performance in the consistency of focal spot size, and the fixed $F / N_{\mathrm{f}}$ variable aperture method is more excellent in the consistency of focusing energy intensity and detection sensitivity.

\section{Dynamic Focusing C-Scan Experiment of Annular Array}

4.1. Specimen and Experimental Setup. The detection of titanium alloy specimen is prepared by additive manufacturing method, which has high attenuation and anisotropy properties [17]. Due to the particularity of manufacturing process, the acoustic wave will produce distortion and strong attenuation when propagating inside the specimen $[18,19]$. Therefore, the focal spot symmetry and focusing acoustic field energy of transducer have great influence on the defect imaging accuracy and SNR, resulting in the poor detection results of additive manufacturing titanium alloy using ultrasonic linear array transducer and single-crystal ultrasonic transducer. The thickness of the specimen along the deposition direction is $60 \mathrm{~mm}$, and there are flat bottom holes with a diameter of $0.8 \mathrm{~mm}$ on three adjacent surfaces to verify the design method of annular array transducer and the variable aperture focusing method, as shown in Figure 8.

An ultrasonic array water immersion C-scan automatic detection system was established to evaluate the defects of additive manufacturing titanium alloy specimen [20], as shown in Figure 9(a). The ultrasonic annular array transducer with a center frequency of $10 \mathrm{MHz}$ and 16 elements is used for the detection experiment. The 64/128 channel ultrasonic array board produced by American AOS company is used as the excitation/receiving hardware of acoustic wave detection. As shown in Figure 9(b), the single detection region along the axis direction is discretized into dynamic focusing points with a certain interval. The dynamic focusing points are set at $20 \mathrm{~mm}, 40 \mathrm{~mm}$, and $60 \mathrm{~mm}$ intervals, which are consistent with the parameters in acoustic field simulation analysis. According to the simulation results of axial acoustic field, the defect- 2 and defect- 3 are located in the nonfocusing region, and the bottom defect- 1 is located in the focusing region of $60 \mathrm{~mm}$. Finally, the 3D finite element simulation model of acoustic beam propagation of annular array in double-layer medium is established, as shown in Figure 9(c). The correctness of the focusing delay time of the annular array transducer in the water coupling automatic scanning is verified, and the propagation of the focusing acoustic beam in the specimen is analyzed.

4.2. Detection Results and Discussion. The detection results of the commonly used linear array are shown in Figure 10(a). Due to the high attenuation and anisotropy of the additive manufacturing titanium alloy, the defect- 1 at the bottom of the specimen cannot be detected by the 64 elements of linear array transducer. In addition, the noncircular symmetric array element distribution structure of linear array will lead to more serious imaging distortion. The $\mathrm{C}$-scan detection results of the annular array are shown in Figure 10(b) and Figure 10(c) by using the dynamic focusing method of fixed aperture and fixed $F / N_{\mathrm{f}}$ variable aperture, respectively [21]. The two focusing methods of annular array can detect all the three defects in the specimen with different depths. However, although the depth of defect- 2 and defect- 3 is smaller, the center amplitude of bottom defect- 1 with fixed aperture focusing method is much larger than that of near surface defect- 2 and defect- 3 , while the center amplitude of defects in variable aperture focusing method is more consistent. In addition, since the variable aperture method is more consistent in the focal spot size at different depths, the size difference between defects in Figure 10(c) is smaller than that of the $\mathrm{C}$-scan results of fixed aperture.

Figure 11 is the echo signals of defects in Figure 10(b) and Figure 10(c), respectively. The detection results show that the SNR and near-field characteristics of the variable aperture method are better than the fixed aperture method. Among them, the improvement of detection results for defect- 2 and defect- 3 in the nonfocusing region is more obvious than defect-1 in the focusing region with variable aperture method. Although the excitation aperture of the variable aperture method is less than that of the fixed aperture method at this depth, the defect center amplitude of the variable aperture method is higher and the overall noise level is relatively low. For example, the SNR of defect- 3 with variable aperture method is $18.1 \mathrm{~dB}$, while that of fixed aperture method is only $9.6 \mathrm{~dB}$. In addition, it can be seen from the A-type signal that the length and amplitude of the nearfield signal using the annular array variable aperture method is far better than that of the fixed aperture method. The nearfield signal length of the fixed aperture method is $7.5 \mathrm{~mm}$, and the maximum amplitude is about $65 \%$ of the full screen; the near-field length of the variable aperture method is 
$3.2 \mathrm{~mm}$, and the maximum amplitude is only $14 \%$ of the full screen.

\section{Summary and Conclusions}

The relationship between the annular array element parameters and the 3-D acoustic field distribution is analyzed, and the parameter design criteria of ultrasonic annular array transducer are established. Different from the design criteria of linear array transducer element size, the annular array transducer can obtain better acoustic field distribution characteristics when the element width is close to the detection wavelength, and the stronger main lobe energy can be achieved with 4 array elements. In addition, when the element width is $\lambda \leq d \leq 2 \lambda$, the high-energy side lobe and grating lobe can be avoided at the same time; when the array element number is $N \geq 8$, and the element width is $0.5 \lambda \leq$ $d \leq \lambda$, the ultrasonic annular array transducer has better axial acoustic field.

The variable dynamic focusing method of ultrasonic annular array transducer is established; compared with the fixed aperture method, the consistency of the focusing point energy and the focusing spot size is higher, and the detection sensitivity of the nonfocusing region is improved. The detection results of additive manufacturing titanium alloy specimen show that the difference of center amplitude and imaging size of different depth defects is smaller by using the variable aperture method. In addition, the SNR of the nonfocusing region defect reaches $\Phi 0.8 \mathrm{~mm}-18.1 \mathrm{~dB}$ and has better near-field characteristics. The proposed method has a good application prospect in the detection of large thickness and high attenuation materials in the whole depth range. The distortion of defect imaging caused by the anisotropy of additive manufacturing titanium alloy will be studied in the next step.

\section{Data Availability}

The data that support the findings of this study are available from the corresponding author upon reasonable request.

\section{Conflicts of Interest}

The authors declare that there is no conflict of interest regarding the publication of this paper.

\section{Acknowledgments}

This work is supported by the National Natural Science Foundation of China (NSFC) No. 11464030.

\section{References}

[1] L. W. Schmerr, Fundamentals of Ultrasonic Phased Arrays, vol. 215 of Solid Mechanics and Its Applications, Springer International Publishing, Cham, 2015.

[2] Y. X. Dai, S. G. Yan, and B. X. Zhang, "Ultrosonic beam steering behavior of linear phased arrays in solid," in 2019 14th Symposium on Piezoelectrcity, Acoustic Waves and Device Applications (SPAWDA), Shijiazhuang, China, China, 2019.
[3] C. J. L. Lane, A. K. Dunhill, B. W. Drinkwater, and P. D. Wilcox, "The inspection of anisotropic single-crystal components using a 2-D ultrasonic array," IEEE Transactions on Ultrasonics, Ferroelectrics and Frequency Control, vol. 57, no. 12, pp. 2742-2752, 2010.

[4] R. J. Housden, A. H. Gee, G. M. Treece, and R. W. Prager, "Ultrasonic imaging of 3D displacement vectors using a simulated 2D array and beamsteering," Ultrasonics, vol. 53, no. 2, pp. 615-621, 2013.

[5] J. G. McKee, R. L. T. Bevan, P. D. Wilcox, and R. E. Malkin, "Volumetric imaging through a doubly-curved surface using a 2D phased array," NDT \& E International, vol. 113, article 102260, 2020.

[6] Z. Zhou, "Development of ultrasonic phased array immersion C-scan automatic detection system," Journal of Mechanical Engineering, vol. 53, no. 12, pp. 28-34, 2017.

[7] J. Woo and Y. Roh, "Design and fabrication of an annular array high intensity focused ultrasound transducer with an optimal electrode pattern," Sensors and Actuators A: Physical, vol. 290, pp. 156-161, 2019.

[8] X. Guan, J. Zhang, E. M. Rasselkorde, W. A. Abbasi, and S. Kevin Zhou, "Material damage diagnosis and characterization for turbine rotors using three-dimensional adaptive ultrasonic NDE data reconstruction techniques," Ultrasonics, vol. 54, no. 2, pp. 516-525, 2014.

[9] P. D. Wilcox, C. Holmes, and B. W. Drinkwater, "Advanced reflector characterization with ultrasonic phased arrays in NDE applications," IEEE Transactions on Ultrasonics, Ferroelectrics, and Frequency Control, vol. 54, no. 8, 2007.

[10] S. R. Kolkoori, M. U. Rahman, P. K. Chinta, M. Ktreutzbruck, M. Rethmeier, and J. Prager, "Ultrasonic field profile evaluation in acoustically inhomogeneous anisotropic materials using 2D ray tracing model: numerical and experimental comparison," Ultrasonics, vol. 53, no. 2, pp. 396-411, 2013.

[11] O. Martinez, L. G. Ullate, and F. Montero, "Analysis of the ultrasonic field radiated by segmented annular arrays," Journal of Computational Acoustics, vol. 9, no. 3, pp. 757-772, 2011.

[12] L. Feng and X. Qian, "Enhanced sizing for surface cracks in welded tubular joints using ultrasonic phased array and image processing," NDT \& E International, vol. 116, article 102334, 2020.

[13] X. Lei, H. Wirdelius, and A. Rosell, "Experimental validation of a phased array probe model in ultrasonic inspection," Ultrasonics, vol. 108, article 106217, 2020.

[14] C. Li, D. Pain, P. D. Wilcox, and B. W. Drinkwater, "Imaging composite material using ultrasonic arrays," NDT \& E International, vol. 53, pp. 8-17, 2013.

[15] Y. Wu, D. Guo, K. Que, B. Chen, and J. Cheng, “Annular phased array dynamic focusing method for large target ultrasonic testing," Ferroelectrics, vol. 459, no. 1, pp. 14-23, 2014.

[16] W. T. Li, Z. G. Zhou, and Y. Li, "Inspection of butt welds for complex surface parts using ultrasonic phased array," Ultrasonics, vol. 96, pp. 75-82, 2019.

[17] S. E. Zeltmann, N. Gupta, N. G. Tsoutsos, M. Maniatakos, J. Rajendran, and R. Karri, "Manufacturing and security challenges in 3D printing," JOM, vol. 68, no. 7, pp. 1872-1881, 2016.

[18] P. Howard, P. Klaassen, N. Kurkcu, and J. Barshinge, "Phased array ultrasonic inspection of titanium forgings," Review of Progress in Quantitative Nondestructive Evaluation, vol. 894, no. 1, pp. 854-861, 2007. 
[19] X. Wang, W. Li, Y. Li et al., "Phased array ultrasonic testing of micro-flaws in additive manufactured titanium block," Materials Research Express, vol. 7, no. 1, article 016572, 2020.

[20] Q. Y. Jiang, X. R. Gao, C. Y. Peng, and J. L. Li, “Application of water immersion ultrasonic phased array technology in wheel rim inspection," Advanced Materials Research, vol. 468-471, pp. 733-737, 2012.

[21] J. Oh, "Phase delay quantization error analysis at a focal plane for an ultrasonic annular arrays imaging system," IEICE Transactions on Fundamentals of Electronics, Communications and Computer Sciences, vol. E90-A, no. 5, pp. 1105-1106, 2007. 